\title{
Functional variation (Q63R) in the cannabinoid CB2 receptor may affect the severity of COVID-19: a human study and molecular docking
}

\author{
Mostafa Rastegar $^{1} \cdot$ Saeed Samadizadeh ${ }^{1} \cdot$ Mohammad Yasaghi $^{1} \cdot$ Abdolvahab Moradi $^{1} \cdot$ Alijan Tabarraei $^{1}$. \\ Vahid Salimi ${ }^{2} \cdot$ Alireza Tahamtan $^{1,3}$ (1)
}

Received: 8 May 2021 / Accepted: 16 July 2021 / Published online: 13 September 2021

(c) The Author(s), under exclusive licence to Springer-Verlag GmbH Austria, part of Springer Nature 2021

\begin{abstract}
Evidence supports a role of host genetic diversity in the clinical course of coronavirus disease 2019 (COVID-19). Variation in the cannabinoid CB2 receptor gene (CNR2) could affect the regulatory action of endocannabinoids on the immune system, resulting in an increased risk of various inflammatory diseases. The present study investigated the relationship between the CNR2-Q63R variant and COVID-19 severity. A total of 200 Iranian COVID-19 patients were enrolled in the study and genotyped using a TaqMan assay. The co-dominant, dominant, recessive, over-dominant, and additive inheritance models were analyzed using SNPStats software. In silico molecular docking was also performed to simulate the effects of the Q63R variation on CB2 binding with a ligand and with the G-protein. A significant difference in the Q63R allele and genotype distribution was found between expired and discharged COVID-19 patients in co-dominant, recessive, and additive inheritance models. The molecular docking results showed that the predicted structure of mutant CB2 (63R type) could not bind to the G-protein in the correct position. The data indicated that the Q63R variation in the CNR2 gene may affect the severity of COVID-19. Identification of genes related to susceptibility and severity of COVID-19 may lead to specific targets for drug repurposing or development.
\end{abstract}

\begin{tabular}{ll}
\multicolumn{2}{l}{ Abbreviations } \\
SARS-CoV-2 & $\begin{array}{l}\text { Severe acute respiratory syndrome corona- } \\
\text { virus 2 }\end{array}$ \\
COVID-19 & Coronavirus disease 2019 \\
EC & Endocannabinoid \\
CB2 & Cannabinoid receptor 2 \\
CNR2 & Cannabinoid CB2 receptor gene \\
RT-PCR & Reverse transcription polymerase chain \\
& reaction \\
HWE & Hardy-Weinberg equilibrium \\
3D & 3-Dimensional \\
PDB & Protein Data Bank \\
OR & Odds ratios \\
\end{tabular}

Handling Editor: Tim Skern.

Alireza Tahamtan

Dr.tahamtan@goums.ac.ir; Alireza.tmn@gmail.com

1 Department of Microbiology, Faculty of Medicine, Golestan University of Medical Sciences, Gorgan, Iran

2 Department of Virology, School of Public Health, Tehran University of Medical Sciences, Tehran, Iran

3 Infectious Diseases Research Center, Golestan University of Medical Sciences, Gorgan, Iran
CI Confidence interval

AIC Akaike information criterion

RMSD Root-mean-square deviation

ACE2 Angiotensin-converting enzyme 2

RSV Respiratory syncytial virus

GPCRs G-protein-coupled receptors

\section{Introduction}

Severe acute respiratory syndrome coronavirus 2 (SARS$\mathrm{CoV}-2$ ) is a newly emerging virus that causes mild-to-severe respiratory disease, which has been named "coronavirus disease 2019" (COVID-19) [1, 2]. All people are susceptible to the virus infection, but there is considerable variation in the course of disease and outcome among infected individuals [3]. While many infected individuals do not experience any symptoms, others proceed to develop COVID-19; however, severe illness and death occur only in a small minority of patients [4]. Although our understanding of SARS-CoV-2 and COVID-19 is still in its infancy, there is now strong evidence supporting a role of host genetic diversity alongside with other host, viral, and environmental factors in the clinical course of disease [5-13]. Host genetic diversity could 
dictate the clinical response to respiratory viruses through susceptibility to viral infection and the propensity for developing harmful pulmonary inflammation [14]. Finding a relationship between host genetics and the clinical outcome of SARS-CoV-2 infection may be necessary for identifying high-risk individuals.

The endocannabinoid (EC) system is a biological system composed of endogenous cannabinoids and their respective receptors, CB1 and CB2 [15]. The system has been identified as a critical endogenous regulator of immune system homeostasis due to its effects on immune cell development, migration, proliferation, and effector functions [16]. Cannabinoids have been proposed for use as immunomodulators to reduce the inflammatory effects of SARS-CoV-2 [17, 18]. Variations in the cannabinoid CB2 receptor gene (CNR2) could affect intracellular signaling and reduce the effects of ECs, which has been associated with an unbalanced immune response and an increased risk of various inflammatory diseases [19-25]. The mammalian CB2 gene is highly conserved in most of the regions, but not at amino acid position 63 [26]. The CB2-Q63R polymorphism is a missense mutation of the second and third bases at codon 63 of the CNR2 gene, which leads to a $\mathrm{Q} / \mathrm{R}$ substitution, causing a different polarization state of the protein [27]. This variation has been shown to affect the response of CB2 to cannabinoids and differently modulate the EC-induced inhibition of lymphocyte proliferation [28]. While evidence has indicated that the mutation does not affect receptor-ligand binding [27], the exact mechanism behind this action is still unknown.

Focusing on the immunopathogenesis of SARS-CoV-2 and the effects of ECs on the immune system, we describe here how variability in the $C N R 2$ gene can conceivably explain variability in COVID-19 clinical phenotype. In addition, in silico molecular docking was performed to simulate the effects of the CB2-Q63R variation on receptor-ligand and receptor-G-protein interactions. The data imply the involvement of the CNR2 gene in the severity of COVID-19 in Iranian patients.

\section{Patients and methods}

\section{Human study}

A total of 200 Iranian COVID-19 patients were included in this study. The case group consisted of 100 expired patients (50 women and 50 men) with a mean age of 62.08 years, and the control group consisted of 100 discharged patients (50 women and 50 men) with a mean age of 54.45 years. All COVID-19 cases were confirmed using a real-time RTPCR assay targeting the SARS-CoV-2 nucleoprotein (N) and ORF1ab genes (Pishtazteb, Iran). A clinical questionnaire was developed for this study and used to collect data from all patients, including gender, age, clinical symptoms, and comorbidities at admission. Nasopharyngeal samples were collected from all patients and divided into two groups according to their disease outcome. The samples were collected until the number of subjects reached 100 (50 women and 50 men) for each group. All of the subjects in this study were from Golestan Province and had the same geographical origin, and none were related.

Genomic DNA was extracted from the collected nasopharyngeal samples using a DNA extraction kit following the manufacturers' instructions (GeneAll, South Korea). Extracted samples were genotyped for the CNR2 rs35761398 (Q63R) variant using a TaqMan assay with commercial primers and probes (Thermo Fisher, USA): 5'CTGTGA AGGTCATAGTCACGCT3' (F primer), 5'CTCTTCTGG GCCTGCTAAGTG3' (R primer), and CAGGTATGAGGG CTTCCGGCGGAG [CC/TT] GGTGGGAGGACAGGA TCAGATAGA [VIC/FAM] (Probes). The reaction conditions were as follows: $95^{\circ} \mathrm{C}$ for $4 \mathrm{~min}$, followed by 50 cycles of $95^{\circ} \mathrm{C}$ for $15 \mathrm{~s}$ and $60^{\circ} \mathrm{C}$ for $90 \mathrm{~s}$. Both PCR and post-PCR allelic discrimination was performed on an ABI PRISM 7300 system (Applied Biosystems, USA). Genotypes of ten percent random samples per group were confirmed by direct PCR sequencing as described previously [20].

The demographic and clinical data were analyzed using SPSS23 software (IBM, Chicago, IL). The Hardy-Weinberg equilibrium (HWE) and differences in allele and genotypic frequencies were calculated using SNPStats software (a web tool for the analysis of association studies: http://bioinfo. iconcologia.net/SNPstats) [29]. Inheritance models such as co-dominant, dominant, recessive, over-dominant, and log-additive were analyzed using the SNPStats software. The power of the test was calculated using G-power 3.1.9.4 software (Universität Kiel, Germany). Odds ratios (OR) and 95\% confidence intervals (CI) were calculated, and $p$-values less than 0.05 were considered statistically significant. The OR was also adjusted by covariates such as age, gender, clinical symptoms, and comorbidities in the logistic regression model.

\section{Molecular docking}

To predict the 3-dimensional (3D) structure of mutant CB2 (63R), the CB2 protein sequence (NCBI Reference Sequence: NP_001832.1) with a change at position 63 was submitted to the I-TASSER server (https://zhanglab. ccmb.med.umich.edu/I-TASSER), which uses a hierarchical approach to protein structure prediction [30]. The best model is selected from the output based on the confidence score (c-score). The Phyre2 server was also used to predict the 3D structure of 63R based on homology (http://www. sbg.bio.ic.ac.uk/phyre2/html/page.cgi?id=index) [31]. The best model based on confidence and identity was selected as 
a homology model. Both models were submitted to ModRefiner (https://zhanglab.ccmb.med.umich.edu/ModRefiner) for atomic-level, high-resolution protein structure refinement and energy minimization [32]. Ramachandran validation was performed using the MolProbity server (http://molprobity. biochem.duke.edu/) for backbone and structure validation of the 3D models [33].

The 3D coordinates of wild-type CB2 (PDB code: 6PT0) were downloaded from the Protein Data Bank (https://www. rcsb.org) in PDB format and opened in UCSF Chimera software [34], and the R chain (CB2) was selected and prepared for docking by removing water, adding polar hydrogens, and adding charges. The same preparation was used for I-TASSER- and Phyre2-built models of mutant CB2. 2-Arachidonoylglycerol (2-AG) was selected as the main endogenous agonist of CB2. The SDF file was downloaded from the PubChem database (https://pubchem.ncbi.nlm.nih. gov/). The 2D SDF file was converted to a 3D PDB file using OpenBabel 2.4.0 [35] and opened in UCSF Chimera.

Following the preparation of receptors and ligands, blind docking (the binding pocket of CB2 is unknown) was performed using Autodock Vina version 1.1.2 [36]. Docking results were analyzed using UCSF Chimera, those with the top scores were selected, and the binding pocket was visualized in Discovery Studio. Protein-protein docking was performed to investigate the interaction between receptors and G-protein using the HDOCK server (http://hdock.phys. hust.edu.cn) [37], which is based on a hybrid algorithm of template-based modeling and ab initio free docking. At first, HDOCK was applied for wild-type CB2 and G-protein, and the same protocol was then carried out for mutant models and the G-protein. Models with the best docking scores were selected and visualized in UCSF Chimera, and the binding energy was predicted using the PRODIGY server (https:// wenmr.science.uu.nl/prodigy) [38].

\section{Results}

\section{Human study}

All patients were COVID-19 confirmed cases and hospitalized in the central hospital for COVID-19 patients in the city of Gorgan (Sayyad Medical and Educational Center). The details of the demographics, gender, age, and clinical data of all cases are presented in Table 1. The age distribution between expired patients (mean, 62.08 years) was significant compared with discharged patients (mean, 54.45 years) $(p<0.05)$. Moreover, the age distribution between female (mean, 63.12 years) and male (mean, 61.04 years) expired patients was significant compared with the discharged subjects (female mean, 55.64 years; male mean, 53.26 years) $(p<0.05)$. In all patients enrolled in the study, the most
Table 1 Demographics, gender, age, and clinical data of subjects ( $\mathrm{n}$ $=200$ )

\begin{tabular}{|c|c|c|c|}
\hline & All patients & Expired & Discharged \\
\hline \multicolumn{4}{|l|}{ Sex } \\
\hline Male, n (\%) & $100(50)$ & $50(25)$ & $50(25)$ \\
\hline Female, n (\%) & $100(50)$ & $50(25)$ & $50(25)$ \\
\hline Sex ratio & $1: 1$ & $1: 1$ & $1: 1$ \\
\hline \multicolumn{4}{|l|}{ Age (years) } \\
\hline Median & 59 & 54 & 63 \\
\hline Mean & 58.27 & 54.45 & 62.08 \\
\hline \multicolumn{4}{|l|}{ Clinical features, n (\%) } \\
\hline Cough & $132(66)$ & $66(66)$ & $66(66)$ \\
\hline Fever or chills & $119(59.5)$ & $62(62)$ & $57(57)$ \\
\hline Dyspnea & $133(66.5)$ & $74(74)$ & $59(59)$ \\
\hline Fatigue & $7(7)$ & $5(5)$ & $2(2)$ \\
\hline Myalgia & $33(16.5)$ & $8(8)$ & $25(25)$ \\
\hline Headache & $12(6)$ & $6(6)$ & $6(6)$ \\
\hline Ageusia and anosmia & $29(14.5)$ & $17(17)$ & $12(12)$ \\
\hline Sore throat & $41(20.5)$ & $20(20)$ & $21(21)$ \\
\hline Nausea or vomiting & $21(10.5)$ & $8(8)$ & $13(13)$ \\
\hline Diarrhea & $14(7)$ & $7(7)$ & $7(7)$ \\
\hline Chest pain & $10(5)$ & $8(8)$ & $2(2)$ \\
\hline \multicolumn{4}{|l|}{ Comorbidities, n (\%) } \\
\hline Diabetes & $38(19)$ & $27(27)$ & $11(11)$ \\
\hline Hypertensive heart disease & $47(23.5)$ & $35(35)$ & $12(12)$ \\
\hline Renal disorders & $6(3)$ & $4(4)$ & $2(2)$ \\
\hline Immunodeficiency & $1(0.5)$ & $1(1)$ & $0(0)$ \\
\hline Chronic respiratory disease & $8(4)$ & $7(7)$ & $1(1)$ \\
\hline Hepatic disorders & $4(2)$ & $2(2)$ & $2(2)$ \\
\hline Chronic neurological diseases & $2(1)$ & $2(2)$ & $0(0)$ \\
\hline
\end{tabular}

frequently observed symptoms were dyspnea $(66.5 \%)$, cough (66\%), fever or chills (59.9\%), sore throat (20.5\%), myalgia $(16.5 \%)$, ageusia and anosmia (14.5\%), nausea or vomiting $(10.5 \%)$, diarrhea (7\%), headache (6\%), chest pain (5\%), and fatigue (3.5\%). The most frequent comorbidities among the patients were hypertensive heart disease (23.5\%), diabetes (19\%), chronic respiratory disease (4\%), renal disorder (3\%), hepatic disorder $(2 \%)$, chronic neurological diseases $(1 \%)$, and immunodeficiency (0.5\%). Importantly, hypertensive heart disease, diabetes, and chronic respiratory disease were significantly more frequent in expired patients than in discharged patients $(p<0.05)$.

The allelic frequencies and the genotype distributions in expired and discharged patients are shown in Table 2. The frequencies of polymorphisms were found to be in HardyWeinberg equilibrium among all subjects in the expired and discharged groups $(p>0.05)$. When logistic regression was used to carry out association analysis after modeling the SNP effects, the SNP showed a significant difference in co-dominant (OR: 3.33, 95\% CI: 1.25-8.88, $p=0.043$ ), 
Table 2 Allele and genotype frequencies of the Q63R polymorphism in subjects ( $\mathrm{n}$ $=200)$

\begin{tabular}{|c|c|c|c|c|c|c|}
\hline \multirow[t]{2}{*}{ Allele } & \multicolumn{2}{|c|}{ All patients } & \multicolumn{2}{|l|}{ Expired } & \multicolumn{2}{|c|}{ Discharged } \\
\hline & Number & Proportion & Number & Proportion & Number & Proportion \\
\hline $\mathrm{R}$ & 253 & 0.63 & 137 & 0.68 & 116 & 0.58 \\
\hline Q & 147 & 0.37 & 63 & 0.32 & 84 & 0.42 \\
\hline \multicolumn{7}{|c|}{ Genotype } \\
\hline $\mathrm{RR}$ & 78 & 0.39 & 44 & 0.44 & 34 & 0.34 \\
\hline QR & 97 & 0.48 & 49 & 0.49 & 48 & 0.48 \\
\hline QQ & 25 & 0.12 & 7 & 0.07 & 18 & 0.18 \\
\hline
\end{tabular}

recessive (OR: $2.92,95 \% \mathrm{CI}: 1.16-7.33, p=0.017$ ), and additive models (OR: $1.62,95 \% \mathrm{CI}: 1.06-2.48, p=0.025$ ) (Table 3). The recessive model was accepted as the best inheritance model to fit the data because of the smaller Akaike information criterion (AIC) value (275.6). A more significant difference was observed after adjusting for covariates (Table 3). No association between CB2-Q63R variants and demographic and clinical features was found (Table 4). It is important to note that the power of the test was 0.90486 .

\section{Molecular docking}

The I-TASSER server predicted five models for the submitted sequences, and we selected the best predicted $3 \mathrm{D}$ structure of mutant CB2 (63R) with c-score, i.e., -0.9 with a $0.74 \mathrm{TM}$-score. The C-score shows the quality of the model predicted by I-TASSER, and a model with a higher c-score signifies a higher confidence level. The TM-score shows the similarity between the predicted model and the template (6PT0), and a TM-score of more than 0.5 exhibits the correct topology for the predicted model. The best model of homology with $100 \%$ confidence and $94 \%$ identity was selected. The 3D structure of the predicted $63 \mathrm{R}$ model and wild-type CB2 (PDB ID 6PT0, Chain R) are shown in Figure 1. Ramachandran plots are shown in Figure 2.

Molecular docking was performed to examine the binding affinity and identify a possible binding pocket of 2-AG with both the newly built CB2-63R models and the wild-type CB2-Q63 structure. The binding energy between 2-AG and CB2-Q63, 2-AG and the I-TASSER-built model, and 2-AG and the Phyre2-built model were -7.5, -6.9, and $-7.0 \mathrm{kcal} /$ mol, respectively. A lower binding energy indicates stronger interaction, higher affinity, and more stability between the ligand and protein. The binding position, hydrogen bonds, alkyl bonds, van der Waals interactions, and binding residues are shown in Figure 3.

The HDOCK server was used for protein-protein docking and predicting the sites of interaction between CB2 and the G-protein. The docking results for wild-type CB2 and G-protein were a docking score of -452.97 and a rootmean-square deviation (RMSD) of $1.06 \AA$, and the binding affinity was $-13.3(\mathrm{kcal} / \mathrm{mol})$. These data showed the correct binding position of the G-protein and CB2-Q63 after docking when compared with the structure of $\mathrm{CB} 2$ bound to the G-protein (PDB ID: 6PT0). The docking results for the I-TASSER model and the G-protein were a docking score of -350.14 and a ligand RMSD of $80.32 \AA$, and for

Table 3 Association of the Q63R polymorphism with COVID-19 severity ( $n=200$, crude analysis) under different inheritance models

\begin{tabular}{|c|c|c|c|c|c|c|c|c|c|}
\hline \multirow[t]{2}{*}{ Model } & \multirow[t]{2}{*}{ Genotype } & \multirow[t]{2}{*}{ Expired n (\%) } & \multirow[t]{2}{*}{ Discharged n (\%) } & OR $(95 \% \mathrm{CI})$ & \multirow[t]{2}{*}{$P$-value } & \multirow[t]{2}{*}{ AIC } & OR $(95 \% \mathrm{CI})$ & $P$-value & \multirow[t]{2}{*}{ AIC } \\
\hline & & & & Not adjusted & & & \multicolumn{2}{|c|}{ Adjusted by covariates* } & \\
\hline \multirow[t]{3}{*}{ Co-dominant } & $\mathrm{RR}$ & $44(44)$ & $34(34)$ & 1.00 & 0.043 & 277 & 1.00 & 0.012 & 254 \\
\hline & QR & $49(49)$ & $48(49)$ & $1.27(0.70-2.31)$ & & & $1.25(0.60-2.62)$ & & \\
\hline & QQ & $7(7)$ & $18(18)$ & $3.33(1.25-8.88)$ & & & $5.48(1.63-18.47)$ & & \\
\hline \multirow[t]{2}{*}{ Dominant } & $\mathrm{RR}$ & $44(44)$ & $34(34)$ & 1.00 & 0.15 & 279.2 & 1.00 & 0.16 & 258.9 \\
\hline & QR-QQ & $56(56)$ & $66(6)$ & $1.53(0.86-2.70)$ & & & $1.66(0.82-3.37)$ & & \\
\hline \multirow[t]{2}{*}{ Recessive } & RR-QR & $93(93)$ & $82(82)$ & 1.00 & 0.017 & 275.6 & 1.00 & 0.0034 & 252.4 \\
\hline & QQ & $7(7)$ & $18(18)$ & $2.92(1.16-7.33)$ & & & $4.81(1.55-14.93)$ & & \\
\hline \multirow[t]{2}{*}{ Over-dominant } & RR-QQ & $51(52)$ & $52(52)$ & 1.00 & 0.89 & 281.2 & 1.00 & 0.54 & 260.6 \\
\hline & QR & 49 (49) & $48(48)$ & $0.96(0.55-1.67)$ & & & $0.81(0.41-1.59)$ & & \\
\hline Log-additive & --- & --- & --- & $1.62(1.06-2.48)$ & 0.025 & 276.3 & $1.93(1.14-3.26)$ & 0.012 & 254.7 \\
\hline
\end{tabular}

OR, odds ratio; 95\% CI, 95\% confidence interval; AIC, Akaike's information criterion

*Covariates are age, gender, and clinical data 
Table 4 Demographics, gender, and clinical form according to Q63R polymorphism

\begin{tabular}{|c|c|c|c|c|c|}
\hline & $\mathrm{RR}$ & $\mathrm{QR}$ & QQ & Total & $P$-value \\
\hline \multicolumn{6}{|l|}{ Gender, n (\%) } \\
\hline Male & $44(56.4)$ & $43(44.3)$ & $13(48)$ & $100(50)$ & \multirow[t]{3}{*}{0.277} \\
\hline Female & $34(43.6)$ & $54(55.7)$ & $12(52)$ & $100(50)$ & \\
\hline Total & $78(100)$ & $97(100)$ & $25(100)$ & $200(100)$ & \\
\hline \multicolumn{6}{|l|}{ Symptoms, n (\%) } \\
\hline Cough & $55(70.5)$ & $60(61.9)$ & $17(68)$ & $132(66)$ & 0.474 \\
\hline Fever or chills & $45(57.7)$ & $57(58.8)$ & $17(68)$ & $119(59.9)$ & 0.645 \\
\hline Dyspnea & $57(73.1)$ & $61(62.9)$ & $15(60)$ & $133(66.5)$ & 0.278 \\
\hline Fatigue & $5(6.4)$ & $2(2.1)$ & $0(0)$ & $7(3.5)$ & 0.178 \\
\hline Myalgia & 13 (16.7) & $17(17.5)$ & $3(12)$ & $33(16.5)$ & 0.801 \\
\hline Headache & $5(6.4)$ & $7(7.2)$ & $0(0)$ & $12(6)$ & 0.392 \\
\hline Ageusia and anosmia & $8(10.3)$ & $19(19.6)$ & $2(8)$ & $29(14.5)$ & 0.135 \\
\hline Sore throat & $15(19.2)$ & $22(22.7)$ & $4(16)$ & $41(20.5)$ & 0.715 \\
\hline Nausea or vomiting & $10(12.8)$ & $11(11.3)$ & $0(0)$ & $21(10.5)$ & 0.178 \\
\hline Diarrhea & $6(7.7)$ & $6(6.2)$ & $2(8)$ & $14(7)$ & 0.907 \\
\hline Chest pain & $2(2.6)$ & $6(6.2)$ & $2(8)$ & $10(5)$ & 0.420 \\
\hline \multicolumn{6}{|l|}{ Comorbidities, n (\%) } \\
\hline Diabetes & 14 (17.9) & $20(20.6)$ & $4(16)$ & 38 (19) & 0.832 \\
\hline Hypertensive heart disease & $17(21.8)$ & $24(24.7)$ & $6(24)$ & $47(23.5)$ & 0.899 \\
\hline Renal disorders & $3(3.8)$ & $3(3.8)$ & $0(0)$ & $6(3)$ & 0.616 \\
\hline Immunodeficiency & $1(1.3)$ & $0(0)$ & $0(0)$ & $1(0.5)$ & 0.456 \\
\hline Chronic respiratory disease & $3(4)$ & $3(3.1)$ & $2(8)$ & $8(4)$ & 0.534 \\
\hline Hepatic disorders & $0(0)$ & $4(4.1)$ & $0(0)$ & $4(2)$ & 0.115 \\
\hline Chronic neurological diseases & $2(2.6)$ & $0(0)$ & $0(0)$ & $2(1)$ & 0.206 \\
\hline
\end{tabular}

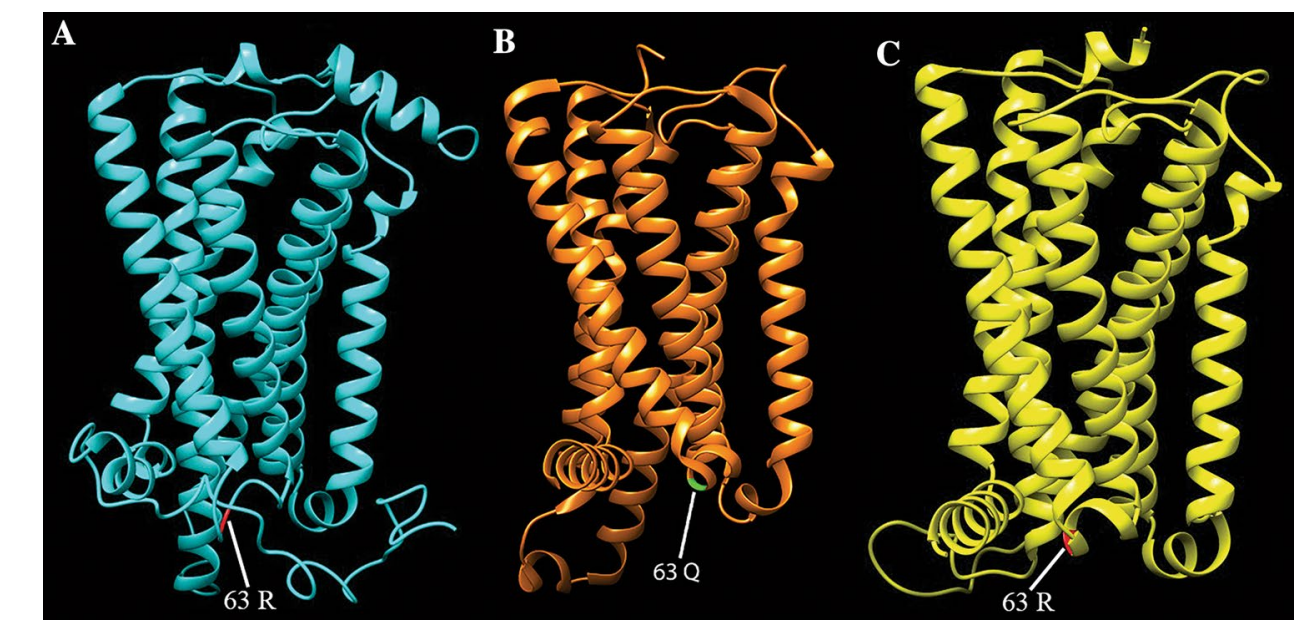

Fig. 1 The 3-dimensional structure of (A) the I-TASSERbuilt model of mutant $\mathrm{CB} 2$ (63R), (B) wild-type CB2 (PDB ID: 6PT0, R chain), and (C) the Phyre2-built model of 63R. The position of amino acid 63 is shown the Phyre 2 model and G-protein, we obtained a docking score of -322.83 and a ligand RMSD of $192.66 \AA$. These results show that neither of the predicted structures of mutant CB2 was able to bind to the G-protein at the correct position. The interaction between both CB2-Q63 and CB2-63R models and the G-protein is shown in Figure 4.

\section{Discussion}

As a newly emerging virus, all people are susceptible to SARS-CoV-2 infection, although the nature and severity of COVID-19 vary significantly among cases. Notably, the reported disease burden and case fatality rates differ 

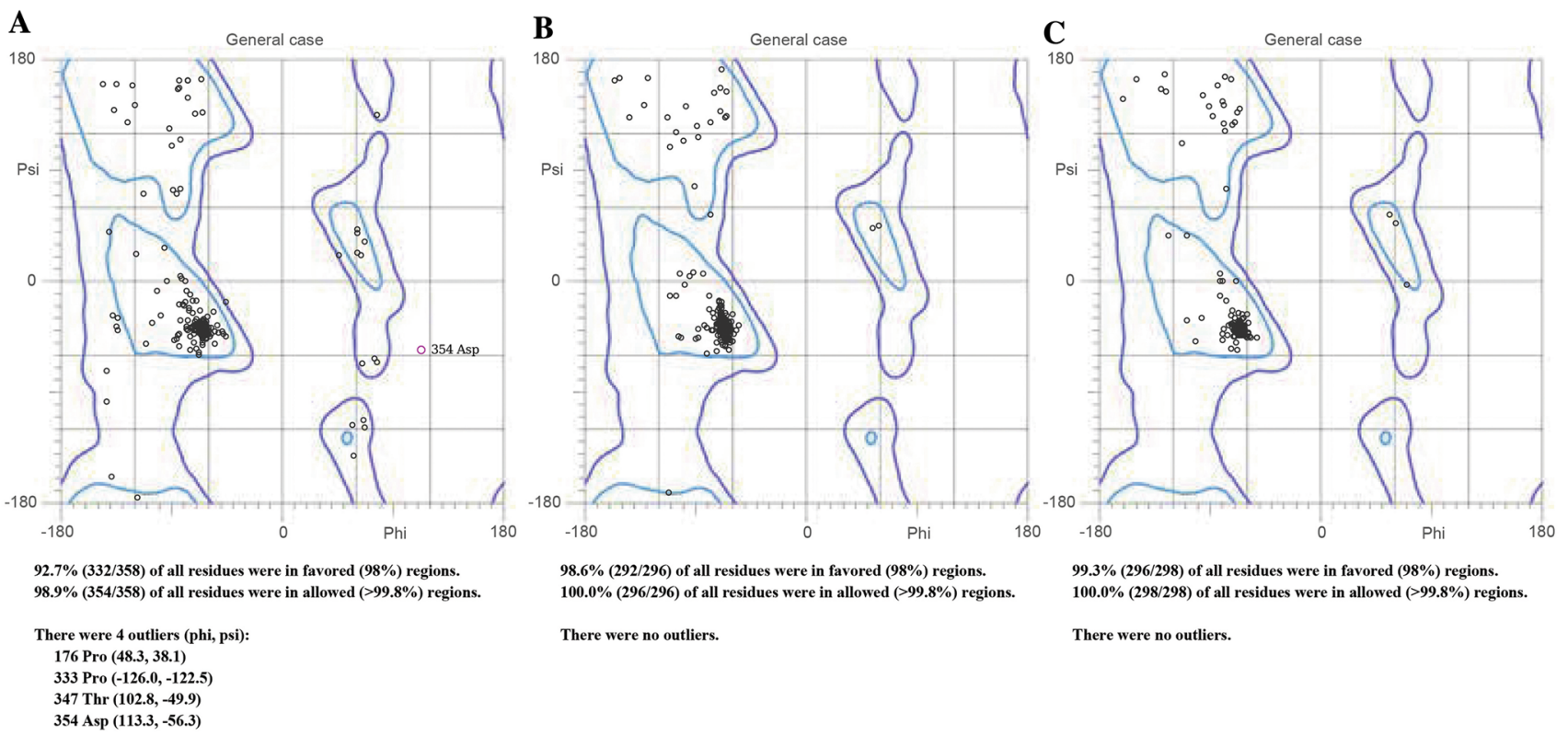

Fig. 2 Ramachandran plots of (A) the I-TASSER-built model of mutant CB2 (63R), (B) wild-type CB2 (PDB ID: 6PT0, R chain), and (C) the Phyre2-built model of 63R. Favored and allowed residues and outliers are shown

considerably from one country to another [39]. COVID19 is known as a multisystemic disease, and many factors influence the disease severity and outcome [13]. The exact influence of host genetic makeup in this variation has remained mostly unknown. The importance of the contribution of host genetics in the differential responses to SARS-CoV-2 is highlighted by a modeling study, revealing that $50 \%$ of the variance of the 'predicted COVID19 ' phenotype is due to genetic factors [6]. For example, studies have shown that the variable expression pattern and genetic variation in angiotensin-converting enzyme 2 (ACE2), a functional receptor for SARS-CoV-2, might be associated with the susceptibility to infection and the severity of the disease [40]. Notably, a recent genomewide association study revealed that critical illness in COVID-19 is related to host antiviral defense mechanisms (IFNAR2 and $O A S$ genes) and mediators of inflammatory organ damage (DPP9, TYK2, and CCR2) [41]. Thus, genes related to immune responses are of particular interest for our understanding of predisposition to severe COVID-19 because of the immunopathology of SARS-CoV-2.

The EC system has received much attention due to its regulatory roles in the immune response and its effects on immune-associated disease progression [42]. There is evidence supporting the system's specific involvement in respiratory-virus-associated immunopathology and in modulating inflammation following infection. We previously provided evidence that the EC system plays an essential role during respiratory syncytial virus (RSV) infection in humans and mice [19]. Other studies have also shown that the lack of cannabinoid receptors increases inflammation and tissue damage following influenza virus infection, and their activation can impair immune responses induced by the virus [43-45]. Such data support the idea of using cannabinoids as a potential therapeutic approach in COVID-19 patients $[17,18]$. The rationale behind the current study was based on SARS-CoV-2 immunopathology, together with the data available on the immune regulatory role of CB2 signaling. As uncontrolled inflammation (known as a cytokine storm) is a hallmark of COVID-19 pathogenesis, investigating factors that affect immune regulation such as the EC system could be helpful.

The CB2-Q63R polymorphism is caused by two missense mutations in the $C N R 2$ gene, changing CAA to CGG, which leads to replacement of an uncharged polar amino acid (glutamine) with a positively charged polar amino acid (arginine) at position 63 in the first intracellular loop of the CB2 receptor [27]. Studies have indicated that the CB2-63R variant has impaired function compared to 63Q in the modulation of immune responses, especially $\mathrm{T}$-cell proliferation [28]. While the exact mechanism is unknown, it has been reported that the signal intensity caused by $63 \mathrm{R}$ activation is weaker than that caused by 63Q activation [46]. The present study shows for the first time an association between the CB2 receptor and COVID-19 severity. A significant difference in the Q63R allele and genotype distribution was found between expired and discharged COVID-19 patients (Table 2). The co-dominant, recessive, and additive inheritance models showed a significant association between Q63R and COVID-19 severity (Table 3). According to the 
Fig. 3 Visualization of docking analysis of 2-arachidonoylglycerol (2-AG) with (A) wild-type CB2, (B) the I-TASSER-built mutant CB2 model, and (C) the Phyre2-built mutant CB2 model. The binding position, hydrogen bonds, alkyl bonds, van der Waals interactions, pi-sigma, carbon, and binding residues are shown

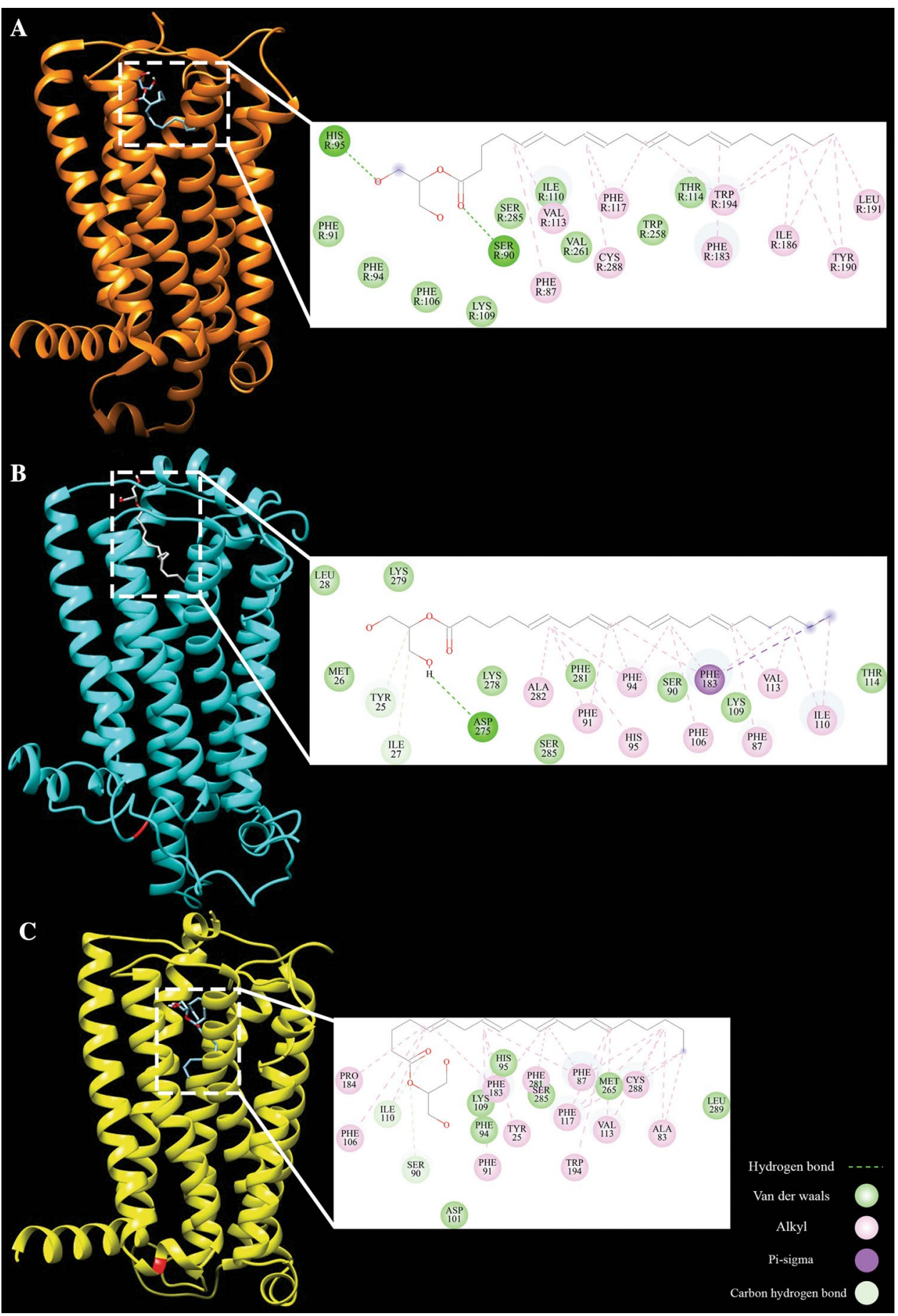

co-dominant model, RR subjects showed more than three times the risk of developing severe COVID-19 than QQ subjects.

An association between the CB2-Q63R variation and autoimmune conditions such as thrombocytopenic purpura [47], celiac disease [25], juvenile idiopathic arthritis [21], inflammatory bowel disease [48], and rheumatoid arthritis [24] has been reported. The data from our previous study suggested an involvement of the Q63R variation in susceptibility to multiple sclerosis in Iranian patients [20]. Interestingly, our previous study showed that the inflammatory response to RSV is inhibited in patients with QQ variants, allowing the virus to replicate and induce severe infection [19]. In the case of SARS-CoV-2 infection, while a robust innate immune response is essential to eliminate viral pathogens, a prolonged, dysregulated, or excessive response can damage the respiratory tract [49]. The current results are consistent with previous studies that showed 
Fig. 4 The HDOCK results and the binding position of the G-protein and (A) wild-type CB2, (B) the I-TASSER-built mutant CB2 model, and $(\mathrm{C})$ the Phyre2-built mutant CB2 model

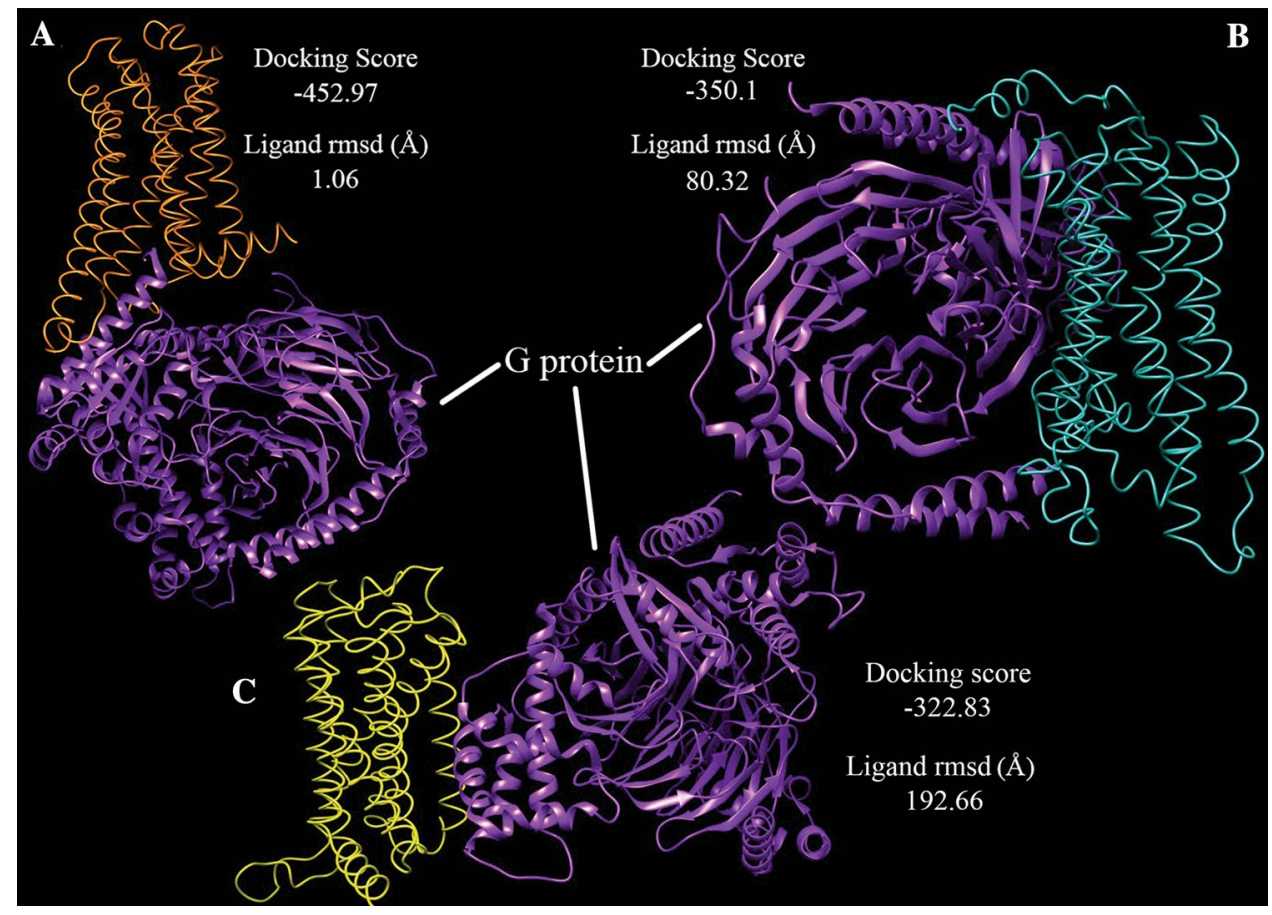

reduced EC-induced modulation of the immune system in human subjects carrying the RR variant of CB2 compared with those with the QQ variant [2, 21, 25, 47, 48].

Importantly, experimental data from a previous competition ligand binding assay showed that the binding affinity of 2-AG for CB2-63R is similar to that for CB2-Q63, but CB2-63R had a significantly lower maximum response after binding to 2-AG compared with the Q63 type [27]. Our molecular docking results confirm that CB2-63R induces a diminished response to ligand binding. The biological effects of cannabinoids are mediated through the activation of G-protein-coupled cannabinoid receptors [50]. G-proteins act as adaptors that link G-proteincoupled receptors (GPCRs) to other signaling and regulatory proteins to operate or modulate intracellular signaling pathways [16]. The molecular docking results show that the predicted structures of mutant $\mathrm{CB} 2$ could not bind to the G-protein at the correct position, resulting in EC signaling dysfunction. These data are consistent with those of Wang et al., who found that CB2-63R induces lower signaling transduction than CB2-63Q in human primary $\mathrm{T}$ cells [46]. The CB2 receptor is predominantly expressed in immune and immune-derived cells, and its activation indirectly affects viral infections by altering host immune responses, particularly inflammation, along different signaling pathways [51-53]. The CB2 Q63R variant modulates the endocannabinoid-induced immune hemostasis differently and consequently results in a lack of balance in immune responses that may turn out to pose a risk of uncontrolled inflammation in COVID-19 patients.

\section{Conclusion}

Data from the current study point toward host genetic involvement in the severity of COVID-19. Host genetics affect the balance of immune responses during viral immunopathogenesis, leading to different clinical phenotypes. The results suggest that people with the CB2-63R variant are more prone to develop severe COVID-19. Thus, the Q63R variation in the CNR2 gene may affect the severity of COVID-19. Considering the potential of this polymorphism as a biomarker for COVID-19 severity, there is an urgent need to deepen these findings through further studies. Regarding the sample size limitation and different genetic backgrounds in various populations, other studies using whole-genome sequencing with a large cohort will be required. Identification of genes related to the susceptibility to and the severity of COVID-19 may lead to specific targets for drug repurposing or development. We hope that, with great effort, scientific support, and information sharing, the overcoming of COVID-19 will come soon.

Acknowledgements The authors would like to thank Golestan University of Medical Sciences and the SARS-CoV-2 laboratory and nursing team who are fighting against the illness. The authors appreciate the financial support of the Research Deputy at Golestan Medical University (111536). This project was extracted from an MSc thesis.

Author's contributions A.T conceptualized and designed the study. M.R, S.S, and M.Y collected samples and did the experiment. A.T and M.R analyzed the collected data. MR drafted the manuscript. A.T, A.M, A.Tb, and V.S evaluated and edited the manuscript. All authors have read and approved the final manuscript. 
Funding No specific funding was obtained for this work.

Data availability The datasets generated and/or analyzed during the current study are available from the corresponding author on reasonable request.

\section{Declarations}

Conflict of interest The authors declare that they have no competing interests.

Ethical approval This study was approved by the ethics committee of the Golestan University of Medical Sciences (IR.GOUMS. REC.1399.005). All procedures were performed in accordance with the relevant guidelines and regulations.

Consent to participate Informed written consent was obtained from all subjects or, if subjects were under 18, from a parent and/or legal guardian.

\section{References}

1. Wang C, Horby PW, Hayden FG, Gao GF (2020) A novel coronavirus outbreak of global health concern. Lancet 395(10223):470-473

2. Chen N, Zhou M, Dong X, Qu J, Gong F, Han Y et al (2020) Epidemiological and clinical characteristics of 99 cases of 2019 novel coronavirus pneumonia in Wuhan, China: a descriptive study. Lancet 395(10223):507-513

3. Teymoori-Rad M, Samadizadeh S, Tabarraei A, Moradi A, Shahbaz MB, Tahamtan A (2020) Ten challenging questions about SARS-CoV-2 and COVID-19. Expert Rev Respir Med 14:881-888

4. Grasselli G, Zangrillo A, Zanella A, Antonelli M, Cabrini L, Castelli A et al (2020) Baseline characteristics and outcomes of 1591 patients infected with SARS-CoV-2 admitted to ICUs of the Lombardy Region Italy. Jama 323(16):1574-1581

5. Sharif-Askari NS, Sharif-Askari FS, Alabed M, Temsah M-H, Al Heialy S, Hamid Q et al (2020) Airways expression of SARSCoV-2 receptor, ACE2, and TMPRSS2 is lower in children than adults and increases with smoking and COPD. Mol Ther Methods Clin Dev 18:1-6

6. Williams FM, Freydin M, Mangino M, Couvreur S, Visconti A, Bowyer RC et al (2020) Self-reported symptoms of covid-19 including symptoms most predictive of SARS-CoV-2 infection, are heritable. medRxiv 6:e1000849

7. Zhao J, Yang Y, Huang H-P, Li D, Gu D-F, Lu X-F et al (2020) Relationship between the ABO blood group and the COVID-19 susceptibility. medRxiv 29:90

8. Korber B, Fischer WM, Gnanakaran S, Yoon H, Theiler J, Abfalterer $\mathrm{W}$ et al (2020) Tracking changes in SARS-CoV-2 spike: evidence that D614G increases infectivity of the COVID-19 virus. Cell. 182(4):812-27.e19

9. Liu Y, Yan L-M, Wan L, Xiang T-X, Le A, Liu J-M et al (2020) Viral dynamics in mild and severe cases of COVID-19. Lancet Infect Dis 20:656-657

10. Tan J, Mu L, Huang J, Yu S, Chen B, Yin J (2005) An initial investigation of the association between the SARS outbreak and weather: with the view of the environmental temperature and its variation. J Epidemiol Community Health 59(3):186-192

11. Lin K, Fong DY-T, Zhu B, Karlberg J (2006) Environmental factors on the SARS epidemic: air temperature, passage of time and multiplicative effect of hospital infection. Epidemiol Infect 134(2):223-30

12. Roxby AC, Greninger AL, Hatfield KM, Lynch JB, Dellit TH, James A et al (2020) Detection of SARS-CoV-2 among residents and staff members of an independent and assisted living community for older adults-Seattle, Washington, 2020. Morb Mortal Wkly Rep 69(14):416

13. Samadizadeh S, Masoudi M, Rastegar M, Salimi V, Shahbaz MB, Tahamtan A (2021) COVID-19: why does disease severity vary among individuals? Respir Med 180:106356

14. Tahamtan A, Samadizadeh S, Rastegar M, Nakstad B, Salimi V (2020) Respiratory syncytial virus infection: why does disease severity vary among individuals? Expert Rev Respir Med 14(4):415-423

15. Lu H-C, Mackie K (2016) An introduction to the endogenous cannabinoid system. Biol Psychiatry 79(7):516-525

16. Tahamtan A, Tavakoli-Yaraki M, Rygiel TP, Mokhtari-Azad T, Salimi V (2016) Effects of cannabinoids and their receptors on viral infections. J Med Virol 88(1):1-12

17. Tahamtan A, Tavakoli-Yaraki M, Salimi V (2020) Opioids/ cannabinoids as a potential therapeutic approach in COVID-19 patients. Expert Rev Respir Med 14(10):965-967

18. Rossi F, Tortora C, Argenziano M, Di Paola A, Punzo F (2020) Cannabinoid receptor type 2: a possible target in SARS-CoV-2 (CoV-19) infection? Int J Mol Sci 21(11):3809

19. Tahamtan A, Samieipoor Y, Nayeri FS, Rahbarimanesh AA, Izadi A, Rashidi-Nezhad A et al (2018) Effects of cannabinoid receptor type 2 in respiratory syncytial virus infection in human subjects and mice. Virulence 9(1):217-230

20. Tahamtan A, Rezaiy S, Samadizadeh S, Moradi A, Tabarraei A, Javid N et al (2020) Cannabinoid CB2 receptor functional variation (Q63R) is associated with multiple sclerosis in Iranian subjects. J Mol Neurosci 70(1):26-31

21. Bellini G, Olivieri AN, Grandone A, Alessio M, Gicchino M, Nobili B et al (2015) Association between cannabinoid receptor type 2 Q63R variant and oligo/polyarticular juvenile idiopathic arthritis. Scand J Rheumatol 44(4):284-287

22. Gouda HM, Kamel NRM (2013) Cannabinoid CB2 receptor gene (CNR2) polymorphism is associated with chronic childhood immune thrombocytopenia in Egypt. Blood Coag Fibrinol 24(3):247-251

23. Strisciuglio C, Bellini G, Miele E, Martinelli M, Cenni S, Tortora $\mathrm{C}$ et al (2018) Cannabinoid receptor 2 functional variant contributes to the risk for pediatric inflammatory bowel disease. J Clin Gastroenterol 52(5):e37-e43

24. Ismail M, Khawaja G (2018) Study of cannabinoid receptor 2 Q63R gene polymorphism in Lebanese patients with rheumatoid arthritis. Clin Rheumatol 37(11):2933-2938

25. Rossi F, Bellini G, Tolone C, Luongo L, Mancusi S, Papparella A et al (2012) The cannabinoid receptor type 2 Q63R variant increases the risk of celiac disease: implication for a novel molecular biomarker and future therapeutic intervention. Pharmacol Res 66(1):88-94

26. Brown SM, Wager-Miller J, Mackie K (2002) Cloning and molecular characterization of the rat $\mathrm{CB} 2$ cannabinoid receptor. Bioch Biophys Acta 1576(3):255-64

27. Carrasquer A, Nebane NM, Williams WM, Song Z-H (2010) Functional consequences of nonsynonymous single nucleotide polymorphisms in the CB2 cannabinoid receptor. Pharmacogenet Genomics 20(3):157-166

28. Sipe JC, Arbour N, Gerber A, Beutler E (2005) Reduced endocannabinoid immune modulation by a common cannabinoid 2 (CB2) receptor gene polymorphism: possible risk for autoimmune disorders. J Leukoc Biol 78(1):231-238 
29. Solé X, Guinó E, Valls J, Iniesta R, Moreno V (2006) SNPStats: a web tool for the analysis of association studies. Bioinformatics 22(15):1928-1929

30. Roy A, Kucukural A, Zhang Y (2010) I-TASSER: a unified platform for automated protein structure and function prediction. Nat Protoc 5(4):725-738

31. Kelley LA, Mezulis S, Yates CM, Wass MN, Sternberg MJE (2015) The Phyre2 web portal for protein modeling, prediction and analysis. Nat Protoc 10(6):845-858

32. Xu D, Zhang Y (2011) Improving the physical realism and structural accuracy of protein models by a two-step atomic-level energy minimization. Biophys J 101(10):2525-2534

33. Ramachandran GN, Ramakrishnan C, Sasisekharan V (1963) Stereochemistry of polypeptide chain configurations. J Mol Biol 7(1):95-99

34. Pettersen EF, Goddard TD, Huang CC, Couch GS, Greenblatt DM, Meng EC et al (2004) UCSF Chimera-a visualization system for exploratory research and analysis. J Comput Chem 25(13):1605-1612

35. O'Boyle NM, Banck M, James CA, Morley C, Vandermeersch T, Hutchison GR (2011) Open babel: an open chemical toolbox. J Cheminform 3(1):33

36. Trott O, Olson AJ (2010) AutoDock Vina: improving the speed and accuracy of docking with a new scoring function, efficient optimization, and multithreading. J Comput Chem 31(2):455-461

37. Yan Y, Zhang D, Zhou P, Li B, Huang S-Y (2017) HDOCK: a web server for protein-protein and protein-DNA/RNA docking based on a hybrid strategy. Nucleic Acids Res 45(W1):W365-W373

38. Xue LC, Rodrigues JP, Kastritis PL, Bonvin AM, Vangone A (2016) PRODIGY: a web server for predicting the binding affinity of protein-protein complexes. Bioinformatics 32(23):3676-3678

39. Karadag E (2020) Increase in COVID-19 cases and case-fatality and case-recovery rates in Europe: a cross-temporal meta-analysis. J Med Virol 92(9):1511-1517

40. Devaux CA, Rolain JM, Raoult D (2020) ACE2 receptor polymorphism: susceptibility to SARS-CoV-2, hypertension, multi-organ failure, and COVID-19 disease outcome. J Microbiol Immunol Infect 53(3):425-435

41. Pairo-Castineira E, Clohisey S, Klaric L, Bretherick AD, Rawlik K, Pasko D et al (2020) Genetic mechanisms of critical illness in Covid-19. Nature 344:807

42. Klein TW, Newton C, Friedman H (1998) Cannabinoid receptors and immunity. Immunol Today 19(8):373-381

43. Karmaus PWF, Chen W, Crawford R, Kaplan BLF, Kaminski NE (2013) $\Delta$ 9-tetrahydrocannabinol impairs the inflammatory response to influenza infection: role of antigen-presenting cells and the cannabinoid receptors 1 and 2. Toxicol Sci 131(2):419-433

44. Buchweitz JP, Karmaus PW, Williams KJ, Harkema JR, Kaminski NE (2008) Targeted deletion of cannabinoid receptors CB1 and $\mathrm{CB} 2$ produced enhanced inflammatory responses to influenza $\mathrm{A} /$ $\mathrm{PR} / 8 / 34$ in the absence and presence of Delta9-tetrahydrocannabinol. J Leukoc Biol 83(3):785-796

45. Karmaus PWF, Chen W, Crawford RB, Harkema JR, Kaplan BLF, Kaminski NE (2011) Deletion of cannabinoid receptors 1 and 2 exacerbates APC function to increase inflammation and cellular immunity during influenza infection. J Leukoc Biol 90(5):983-995

46. Wang J, Xu J, Liu J, Zhu H, Peng Y, Ding Z-M et al (2018) Genetic variant Q63R of cannabinoid receptor 2 causes differential ERK phosphorylation in human immune cells. Genet Test Mol Biomarkers 22(5):320-326

47. Rossi F, Mancusi S, Bellini G, Roberti D, Punzo F, Vetrella S et al (2011) CNR2 functional variant (Q63R) influences childhood immune thrombocytopenic purpura. Haematologica 96(12):1883-1885

48. Strisciuglio C, Bellini G, Miele E, Martinelli M, Cenni S, Tortora $C$ et al (2016) Cannabinoid receptor 2 functional variant contributes to the risk of pediatric inflammatory bowel disease. Digest Liver Dis 48:e269

49. Conti P, Ronconi G, Caraffa A, Gallenga CE, Ross R, Frydas I et al (2020) Induction of pro-inflammatory cytokines (IL-1 and IL-6) and lung inflammation by Coronavirus-19 (COVI-19 or SARS-CoV-2): anti-inflammatory strategies. J Biol Regul Homeost Agents 34(2):327-331

50. Piomelli D (2003) The molecular logic of endocannabinoid signalling. Nat Rev Neurosci 4(11):873-884

51. Yao B, Mackie K (2009) Endocannabinoid receptor pharmacology. Curr Top Behav Neurosci 1:37-63

52. Rieder SA, Chauhan A, Singh U, Nagarkatti M, Nagarkatti $P$ (2010) Cannabinoid-induced apoptosis in immune cells as a pathway to immunosuppression. Immunobiology 215(8):598-605

53. Tahamtan A, Tavakoli-Yaraki M, Shadab A, Rezaei F, Marashi SM, Shokri F et al (2018) The role of cannabinoid receptor 1 in the immunopathology of respiratory syncytial virus. Viral Immunol 31(4):292-298. https://doi.org/10.1089/vim.2017.0098

Publisher's Note Springer Nature remains neutral with regard to jurisdictional claims in published maps and institutional affiliations. 